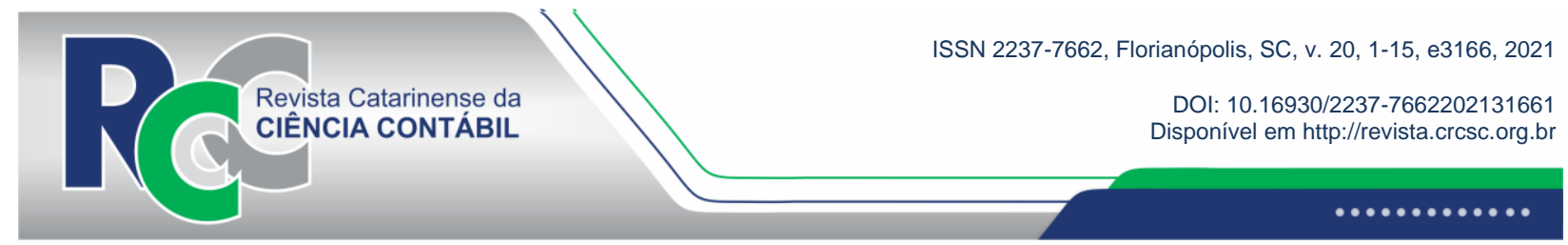

\title{
METACOGNIÇÃO, PERFIL EMPREENDEDOR E PERCEPÇÃO DE SUCESSO
}

\section{METACOGNITION, ENTREPRENEURIAL PROFILE, AND PERCEPTION OF SUCCESS}

\author{
PEDRO MARLUS CAVALCANTE DE \\ ALBUQUERQUE ESTRELA \\ Universidade Federal de Sergipe. Endereço: Rua Alcino de \\ Oliveira Neto, 45 | Luzia | 49045-800 | Aracaju/SE | Brasil. \\ (1) https://orcid.org/0000-0002-3986-7467 \\ pedroestrelaufs@gmail.com
}

\author{
ADRIANO LEAL BRUNI \\ Universidade Federal da Bahia. Endereço: Av. Reitor Miguel \\ Calmon, s/n | Vale do Canela | 40110-903 | Salvador/BA | \\ Brasil. \\ Dhttps://orcid.org/0000-0003-0283-2778 \\ albruni@ufba.br
}

\section{RAIMUNDO NONATO LIMA FILHO}

Universidade de Pernambuco. Autarquia Educacional do Vale do São Francisco. Endereço: Cidade Universitária, s/n, Vila Eduardo | 56.328-903 | Petrolina/PE | Brasil.

Dhttps://orcid.org/0000-0003-4953-5260

rnlfilho@gmail.com

\section{RESUMO}

Este estudo buscou identificar em que medida a metacognição exerce efeito sobre a percepção de sucesso, mediada pelo perfil empreendedor. A amostra analisada envolveu 194 egressos dos cursos de Administração, Ciências Contábeis e Economia de instituições de ensino superior públicas e privadas do estado de Sergipe. Os procedimentos envolveram o uso de modelagem de equações estruturais e os resultados indicaram que a orientação de objetivos apresenta relação significativa direta e indireta com a percepção de sucesso. O conhecimento metacognitivo e a experiência metacognitiva possuem relações indiretas significativas com a percepção de sucesso, e esta relação ocorre com as dimensões "autorrealização" e "líder" do perfil empreendedor. Como contribuição deste estudo, identificaram-se características metacognitivas que podem ser aperfeiçoadas para melhorar a percepção de sucesso, como, por exemplo: definir frequentemente objetivos; compreender a relação entre tarefas e objetivos; estabelecer objetivos específicos; avaliar com frequência o progresso de uma tarefa; selecionar a melhor opção para a resolução de

Editado em português e inglês. Versão original em Português.

Recebido em 23/3/2021. Revisado em 19/5/2021. Aceito em 10/6/2021 pelos Prof. Dr. Sérgio Murilo Petri (Editor-Chefe) e Prof. Dr. Sandro Vieira Soares (Editor Adjunto). Publicado em 30/7/2021.

Copyright (C) 2021 RCCC. Todos os direitos reservados. É permitida a citação de parte de artigos sem autorização prévia, desde que identificada a fonte. 
problemas; usar estratégias assertivas no passado; obter conhecimento prévio de uma tarefa; fragmentar problemas em pequenas partes; pensar antes de realizar uma tarefa; usar diferentes estratégias; organizar o tempo e informações; selecionar informações importantes; e utilizar a intuição para formular estratégias.

Palavras-chaves: Metacognição. Perfil Empreendedor. Percepção de Sucesso. Modelagem de Equações Estruturais. Survey.

\begin{abstract}
This study sought to identify to what extent metacognition has an effect on perceived success, mediated by the entrepreneurial profile. The sample we analyzed involved 194 alumni from Business Administration, Accounting, and Economics programs from public and private higher education institutions in the state of Sergipe. The procedures involved the use of structural equation modeling and the results indicated that goal orientation has a significant direct and indirect relationship with perceived success. Metacognitive knowledge and metacognitive experience have significant indirect relationships with perceived success, and this relationship takes place with the "self-realization" and "leader" dimensions of the entrepreneurial profile. As this study's contribution, we identified metacognitive characteristics that can be improved to enhance the perception of success, such as: frequently defining goals, understanding the relationship between tasks and goals, setting specific goals, frequently evaluating a task's progress, selecting the best option for problem solving, using assertive strategies in the past, gaining prior knowledge regarding a task, breaking problems into small parts, thinking before performing a task, using different strategies, organizing time and information, selecting important information, and using intuition to formulate strategies.
\end{abstract}

Keywords: Metacognition. Entrepreneurial Profile. Perception of Success. Structural Equation Modeling. Survey.

\title{
1 INTRODUÇÃO
}

Estudos envolvendo o empreendedorismo têm aumentado consideravelmente nos últimos trinta anos e ganhado contribuições de diversas áreas e pesquisadores, apresentando novas perspectivas teóricas (Ferreira, Pinto \& Miranda, 2015). Algumas pesquisas buscaram compreender como se dá o sucesso ou por que algumas pessoas têm maior facilidade em detectar oportunidades, tomar decisões e explorá-las.

Lima Filho e Bruni (2015) afirmaram que no momento da tomada de decisão, há o ajuizamento do nosso próprio pensamento, bem como a avaliação de nossas escolhas. Entretanto, conforme os autores, o ato de empreender também exige escolhas que oferecem ao indivíduo a possibilidade de avaliar as decisões. Nesse processo, surge a capacidade metacognitiva.

A metacognição é um tema atual e palpitante que se articula com diversas áreas do conhecimento humano, como a psicologia, a educação, a sociologia, a matemática, a economia, a contabilidade, a administração, dentre outras. A metacognição pode transformar o conhecimento em conduta profissional, uma vez que pensar sobre o próprio pensamento e estabelecer estratégias ampliam as potencialidades individuais em prol da resolução de problemas (Lima Filho \& Bruni, 2015), o que por consequência pode aumentar a percepção de sucesso. Estudos recentes alicerçam a proposta deste estudo ao confirmar relação entre metacognição e percepção de sucesso (Earley \& Ang, 2003; Haynie, Shepherd, Mosakowski \& Earley, 2010; Cho \& Jung, 2014). Algumas pesquisas também associam o perfil empreendedor com a metacognição e a percepção de sucesso (Lima Filho, 2013; Cho \& Jung, 2014). 
Nesse sentido, este artigo parte do pressuposto de que há uma relação positiva significativa entre metacognição e percepção de sucesso, a qual é mediada pelo perfil empreendedor, ou seja, a variável mediadora modifica a relação entre as variáveis independente e dependente.

A partir desse contexto, pretende-se responder ao seguinte problema de pesquisa: em que medida a metacognição exerce efeito sobre a percepção de sucesso, mediada pelo perfil empreendedor?

Justifica-se este estudo não só pela contribuição teórica, mas também por apresentar evidências empíricas que auxiliam a prática de profissionais, estudiosos e empreendedores, ao examinar a relação positiva entre metacognição e percepção de sucesso. Este artigo promove uma melhoria na estratégia reguladora da aprendizagem e apresenta as características metacognitivas que afetam a percepção de sucesso, a qual pode proporcionar aperfeiçoamento e aprendizagem dessas características, além de promover uma melhoria na percepção de sucesso.

\section{FUNDAMENTAÇÃO TEÓRICA}

O empreendedorismo é um amplo campo de estudo da administração que tem avançado em diferentes áreas do conhecimento. Entretanto, o desenvolvimento do conhecimento nessa área não tem ocorrido na velocidade almejada, uma vez que ela engloba inúmeros temas (Ferreira et al., 2015; Ferreira, Colares, Rocha \& Carvalho Junior, 2013).

Um dos temas do empreendedorismo está relacionado à continuidade ou descontinuidade de um negócio e é passível de observação. No entanto, o conceito de percepção de sucesso é menos perceptível. Logo, a sua observação pode ser mais difícil, já que a percepção de sucesso está atrelada ao indivíduo, já que se leva em consideração aspectos psicológicos e subjetivos.

Reijonen e Kompula (2007) mencionam que aspectos como educação familiar, classe social, escolaridade, gênero e experiências podem influenciar a percepção de sucesso do indivíduo. Nesse sentido, Earley e Ang (2003) defendem que a consciência metacognitiva ajuda a identificar estratégias que maximizem as chances de alcançar objetivos, ou seja, a consciência metacognitiva pode influenciar na percepção de sucesso do indivíduo.

Neste estudo, adotam-se três constructos que conduzem os rumos da discussão teórica e da análise empírica: metacognição, perfil empreendedor e percepção de sucesso.

A metacognição é um termo cunhado por Flavell (1979), que a definiu como o conhecimento sobre fenômenos cognitivos, ou seja, a capacidade de pensar sobre o próprio pensamento. Rocha e Malheiro (2019) argumentam que a metacognição é um termo polissêmico e não visível. Rosa, Corrêa, Passos e Arruda (2020) dizem que a metacognição tem relação com a noção de pensamento e o próprio pensamento. A descrição mais comum para a metacognição é o conhecimento e regulação do próprio sistema cognitivo (Lima filho, 2013). Na mesma linha de raciocínio, Haynie e Shepherd (2009) definiram a metacognição como a consciência e a compreensão de seu próprio processo cognitivo. Gewehr, Strohschoen e Schuck (2020) afirmam que "a metacognição é a reflexão da própria cognição, a tomada de consciência do sujeito sobre seus conhecimentos, aprendizagens e limitações".

A metacognição pode ser entendida como um conjunto de habilidades do pensamento para resolver problemas, tornando-se indispensável em ambientes dinâmicos e incertos (Haynie et al., 2010). Ribeiro (2003, p. 110) menciona que a metacognição "diz respeito, entre outras coisas, ao conhecimento do próprio conhecimento, à avaliação, à regulação e à organização dos próprios processos cognitivos". Já Botelho e Vargas (2021) definem a metacognição como uma habilidade humana relacionada ao autoconhecimento e gerenciamento de atividades a serem desenvolvidas. 
A metacognição pode ser dividida em cinco dimensões teóricas: orientação de objetivos, conhecimento metacognitivo, experiência metacognitiva, escolha metacognitiva e monitoramento metacognitivo (Haynie \& Shepherd, 2009; Cho \& Jung, 2014). A segregação da metacognição em dimensões possibilita conhecer quais características metacognitivas relacionam-se mais significativa com a percepção de sucesso, o que pode facilitar o aprimoramento e a aprendizagem de tais características.

A orientação de objetivos, conforme Hirst, Knippenberg e Zhou (2009), refletem as crenças de autodesenvolvimento e a forma como elas direcionam os indivíduos a interpretar e se empenhar no seu ambiente. Ela pode ser dividida em orientação de domínio, que sugere que esforços acarretam melhoria de resultados e que a habilidade é maleável; e orientação de desempenho, na qual a habilidade é demonstrada por desempenhos melhores que outros (Jones, Davis \& Thomas, 2017).

O conhecimento metacognitivo diz respeito à compreensão consciente de questões cognitivas e como estas se relacionam com pessoas, tarefas e estratégias (Flavell, 1987). Tanto Haynie e Shepherd (2009) quanto Deffendi e Schelini (2016) mencionam que o conhecimento metacognitivo é a extensão do que as pessoas sabem sobre si mesmas, outras pessoas, tarefas e estratégias, quando são envolvidas num processo de criação de uma estrutura de múltiplas decisões focadas em interpretar, planejar e implementar objetivos num ambiente de mudanças.

O conhecimento metacognitivo permite avaliar resultados finais ou intermediários e reforçar a estratégia escolhida ou alterá-la, em função das avaliações feitas (Ribeiro, 2003). Ele é armazenado numa base mental e é possível compreendê-lo, adicioná-lo, excluí-lo ou revisá-lo a qualquer momento (Pimentel \& Sales Júnior, 2021).

As vivências metacognitivas são experiências afetivas em que situações anteriores, memórias, intuições e emoções são empregadas como recursos, podendo dar sentido a um contexto de decisão (Flavell, 1987). Haynie e Shepherd (2009) argumentam que experiência passadas, emoções e intuições relacionadas a eventos anteriores podem servir como estrutura de decisão focada em novas decisões.

Deffendi e Schelini (2016) ainda apontam que a experiência metacognitiva tem o objetivo de informar ao indivíduo sobre seu avanço numa determinada tarefa ou atividade, bem como dificuldades e a evolução obtida no processo, além de mostrar o que é possível realizar.

A escolha ou estratégia metacognitiva ocorre quando o indivíduo se envolve num processo de seleção de múltiplas decisões, buscando a melhor maneira de interpretar, planejar e implementar uma resposta, no intuito de gerenciar uma mudança de ambiente (Haynie \& Shepherd, 2009). Haynie et al. (2010) afirmam que essa dimensão se refere à estrutura formulada pelo empreendedor e serve para avaliar múltiplas respostas e alternativas ao processamento da tarefa empreendedora.

Já o monitoramento metacognitivo permite ao indivíduo o processo, observação, reflexão e experimentação de seus próprios processos cognitivos (Flavell, 1979). O monitoramento é a busca do feedback, a fim de reavaliar as outras dimensões metacognitivas, com o objetivo de gerenciar uma mudança de ambiente (Flavell, 1979; Haynie \& Shepherd, 2009). O monitoramento é uma avaliação das decisões tomadas, com o objetivo de melhorar o resultado (Estrela, 2020).

Outro constructo crucial neste trabalho é o perfil empreendedor. Lima Filho e Bruni (2015) destacam que a educação em empreendedorismo pode ser entendida como um caminho que estimula o espírito empreendedor e o comportamento das pessoas Moreira, Alves, Andreassi, \& Braga, (2020). Ferreira et al. (2015) argumentam que a pesquisa sobre esse tema tem crescido nos últimos trinta anos e tem atraído mais atenção ao longo do tempo.

Oosterbeek, Praag e Ijsselstein (2010) mencionam que um indivíduo com um nível mais elevado de concentração de características e competências empreendedoras tem sido usado para 
comparar grupos de indivíduos com perfil empreendedor mais elevado com outros grupos pesquisados.

Schmidt e Bohnenberger (2009) criaram uma estrutura com características do perfil empreendedor: a) autoeficaz; b) assume riscos; c) planejador; d) detecta oportunidades; e) persistente; f) sociável; g) inovador; e h) liderança. Essa pesquisa trata o perfil empreendedor como mediador na relação entre metacognição e percepção de sucesso. Para justificar esse papel, Lima Filho e Bruni (2015) destacam que o empreendedor pode utilizar qualquer estratégia e que a consciência metacognitiva identifica estratégias metacognitivas que maximizem a probabilidade de alcançar um objetivo.

O outro constructo-chave deste trabalho é a percepção de sucesso. Existe uma disparidade de definições sobre sucesso por causa de suas inúmeras formas de medição, mas, embora alguns pesquisadores tentem estudar as condições para alcançar o sucesso, eles não oferecem conclusões unânimes, fazendo com que o fator determinante do sucesso continue sendo uma área intrigante e inexplorada (Staniewsky \& Awruk, 2017).

Existem dois caminhos para avaliar o sucesso: um voltado para o lado pessoal do empreendedor e outro voltado aos indicadores econômicos e financeiros do empreendimento. Neste artigo foram utilizados indicadores que tratam da personalidade e de habilidades, padrões comportamentais e atitudes específicas do indivíduo. Isso se justifica porque as dimensões da metacognição levantam questões de caráter pessoal.

Para compreender o conceito de sucesso, é necessário ter uma percepção subjetiva, levando em conta fatores como satisfação, autonomia e equilíbrio entre trabalho e família (Reijonen \& Komppula, 2007). Isso significa que o sucesso pode ser entendido como o conjunto das realizações profissionais e pessoais.

Costa (2013) acrescenta o lado pessoal do sucesso. Ele argumenta que o sucesso está relacionado a resultados financeiros, promoções, capacidade de adaptação, identidade no trabalho, visibilidade, equilíbrio vida-trabalho, realização pessoal, sentimento de orgulho e reconhecimento.

Dries, Pepermans e Carlier (2008) propuseram um modelo multidimensional na carreira, dividido em quatro quadrantes. Costa e Dutra (2011) explicam que o quadrante I corresponde às realizações interpessoais, como desempenho, promoção e contribuição real; o quadrante II refere-se a realizações intrapessoais e contém duas regiões: desenvolvimento e criatividade; o quadrante III refere-se a sentimentos intrapessoais, relacionados a si mesmo, e contém duas regiões de significado: segurança e satisfação; já o quadrante IV relaciona-se a sentimentos interpessoais, atrelados à relação com terceiros: reconhecimento, que se refere ao sentimento de recompensa por seus esforços, cooperação e contribuição percebida.

\section{PROCEDIMENTOS METODOLÓGICOS}

Este estudo é confirmatório e, conforme Long (1983), partindo da premissa de que existe uma teoria sobre a medição das variáveis em relação aos fatores, busca a confirmação de uma teoria hipotetizada. Enquadra-se no método hipotético-dedutivo, com caráter quantitativo.

A estratégia de coleta de dados utilizada foi um survey, do tipo explanatório, ou seja, dando garantia do anonimato à amostra, já que esse método garante aos participantes confidencialidade e privacidade, encorajando-os a fornecer informações mais fidedignas.

Para o teste das relações das variáveis, bem como para o teste do modelo escolhido, este estudo utiliza o modelo de equações estruturais estimado por mínimos quadrados parciais (MEEPLS). Para a análise dos dados foi utilizado o software R Core Team 2019 - pacote matrixpls. 
Com o objetivo de responder ao problema de pesquisa, foram propostas seis hipóteses. As cinco primeiras buscam relacionar as dimensões da metacognição com a percepção de sucesso. Já a sexta hipótese visa mensurar o efeito mediador do perfil empreendedor na relação entre metacognição e percepção de sucesso. As seis hipóteses são:

$\mathrm{H}_{1}$ - A orientação de objetivos influencia positivamente a percepção de sucesso;

$\mathrm{H}_{2}-\mathrm{O}$ conhecimento metacognitivo influencia positivamente a percepção de sucesso;

$\mathrm{H}_{3}$ - A experiência metacognitiva influencia positivamente a percepção de sucesso;

$\mathrm{H}_{4}$ - A escolha metacognitiva influencia positivamente a percepção de sucesso;

$\mathrm{H}_{5}$ - O monitoramento metacognitivo influencia positivamente a percepção de sucesso;

$\mathrm{H}_{6}$ - O perfil empreendedor medeia a relação entre metacognição e a percepção de sucesso.

A primeira hipótese está alicerçada nas pesquisas de Cho e Jung (2014) e Haynie e Shepherd (2009); a segunda está vinculada aos estudos de Haynie e Shepherd (2009), Earley e Ang (2003) e Urban (2012); a terceira (H3) está conectada aos trabalhos de Haynie et al. (2010) e Flavell (1987); a quarta (H4) está suportada pelos trabalhos de Urban (2012) e Haynie e Shepherd (2009); já a quinta hipótese (H5) ancora-se no estudo de Haynie et al. (2010). Por fim, em relação à sexta hipótese (H6), não foram encontrados trabalhos que comprovassem o efeito mediador do perfil empreendedor na relação entre metacognição e percepção de sucesso, portanto essa é uma contribuição teórico-empírica que este artigo pretende promover.

Os instrumentos de coleta de dados foram compostos por questões fechadas, baseadas em escalas do tipo de Likert com cinco pontos. Os participantes só puderam responder a uma única alternativa por questão em todo o questionário, com exceção do bloco socioeconômico.

Para os itens relacionados à metacognição, foi utilizado o estudo de Haynie e Shepherd (2009). O questionário foi dividido em cinco dimensões metacognitivas.

Para as questões referentes ao perfil empreendedor, foi adotado o questionário desenvolvido por Schimidt e Bohnenberger (2009), que divide o instrumento em seis blocos: a) autorrealização; b) líder; c) planejador; d) inovador; e) assume riscos; e f) sociável.

No que tange à mensuração da percepção de sucesso, foi utilizada uma escala elaborada por Costa (2013), que utilizou o modelo multidimensional de Dries et al. (2008).

A coleta de dados foi realizada a partir da aplicação do questionário eletrônico via Survey Monkey, disponibilizado por e-mail ou via aplicativo de mensagem.

Os respondentes da pesquisa são egressos dos cursos de Administração, Ciências Contábeis e Economia de instituições públicas e privadas do estado de Sergipe, por critério de acessibilidade. Foram selecionados esses cursos porque eles podem apresentar perfis empreendedores diferentes - formais, não formais ou não empreendedores -, além de serem cursos voltados para a área da gestão.

Em relação ao tamanho da amostra, foi utilizado o modelo da raiz quadrada inversa de Kock e Hadaya (2016):

$$
\widehat{N}>\left(\frac{z_{0.95}+z_{0.80}}{|\beta|_{\min }}\right)^{2}
$$

Onde $\mathrm{N}$ é o tamanho da amostra mínimo, $\mathrm{Z}_{0,95}$ é o escore da distribuição normal padrão associado ao nível de confiança adotado (95\%), $\mathrm{Z}_{0,80}$ é o escore da distribuição normal padrão associado ao poder de teste adotado $(80 \%)$ e $\beta_{\min }$ é o menor coeficiente de caminho observado $(0,18)$, ou seja, representa o valor absoluto do coeficiente do caminho estatisticamente significativo com a magnitude mínima no modelo.

Assim, fixamos o tamanho mínimo da amostra em 191 participantes. Entretanto, foram obtidas 225 respostas e, após a exclusão de respostas de questionários invalidados, restou o total de 194 respostas. 
O modelo de mensuração deste estudo é reflexivo, pois, conforme Hair, Hult, Ringle, Sarstedt e Thiele (2017), assume-se que os itens de cada constructo são manifestações do constructo adjacente. Portanto, a avaliação do modelo de mensuração é composta por três pressupostos utilizados em modelos reflexivos: consistência interna, validade convergente e validade discriminante (Hair et al., 2017).

A medida mais comum utilizada para a consistência interna é o Alpha de Cronbach, mas este é sensível ao número de itens na escala (Dijkstra \& Henseler, 2015; Lima Filho \& Nova, 2020). Logo, adotou-se a confiabilidade composta, ou composite reliability (CR), que varia de 0 a 1 , onde valores elevados indicam maior consistência interna.

$\mathrm{Na}$ validade convergente - que, conforme Cheah, Sarstedt, Ringle, Ramayah e Ting (2018), consiste na intensidade com que um medida se relaciona de forma positiva com medidas alternativas do constructo -, foi utilizada a variância média extraída, ou average variance extracted (AVE), e as cargas fatoriais dos itens. Valores de AVE superiores a 50\% indicam que o constructo é unidimensional e possui validade convergente. Entretanto, a raiz quadrada da carga fatorial de um item indica o quanto de sua variação é explicado pelo constructo e precisa ser superior a 0,5 , indicando que as cargas fatoriais devem ser superiores a $0,708(\sqrt{0,5})$.

Contudo, se forem encontradas cargas fatoriais entre 0,4 e 0,7 , deve-se remover itens com cautela. O item só deve ser excluído nessa faixa se sua remoção amplia para níveis favoráveis de CR e AVE. Caso contrário, mantém-se o item (Cheah et al., 2018).

A validade discriminante é o quanto cada dimensão é única, ou seja, diferente das outras dimensões (Hair et al., 2017). Para avaliar a validade discriminante, utilizou-se o critério de heterotrait-monotrait ratio (HTMT). Henseler, Ringle e Sarstedt (2015) sugerem dois pontos de corte para o HTMT: 0,85, quando a teoria é bem estabelecida e 0,90 , quando é uma teoria em desenvolvimento.

Após a validade da consistência dos constructos, procedeu-se a análise do modelo estrutural, ou seja, avaliação das capacidades preditivas e das relações entre os constructos. Os principais critérios utilizados foram a significância dos coeficientes de caminho entre os constructos $\left(\mathrm{R}^{2}\right)$, o tamanho do efeito $\left(\mathrm{f}^{2}\right)$, a relevância preditiva $\left(\mathrm{Q}^{2}\right)$ e o tamanho do efeito $\left(\mathrm{q}^{2}\right)$ (Hair et al., 2017). Os coeficientes são padronizados dentro do intervalo -1 a 1, onde valores próximos a $|1|$ indicam relações fortes e valores próximos a 0 indicam relações fracas. Valores positivos indicam relação direta e valores negativos indicam relação indireta (Hair et al., 2017).

O processamento do modelo de pesquisa envolvendo as relações entre os constructos e suas dimensões por meio da técnica do MEE-PLS resultou no modelo de pesquisa apresentado na Figura 1. 


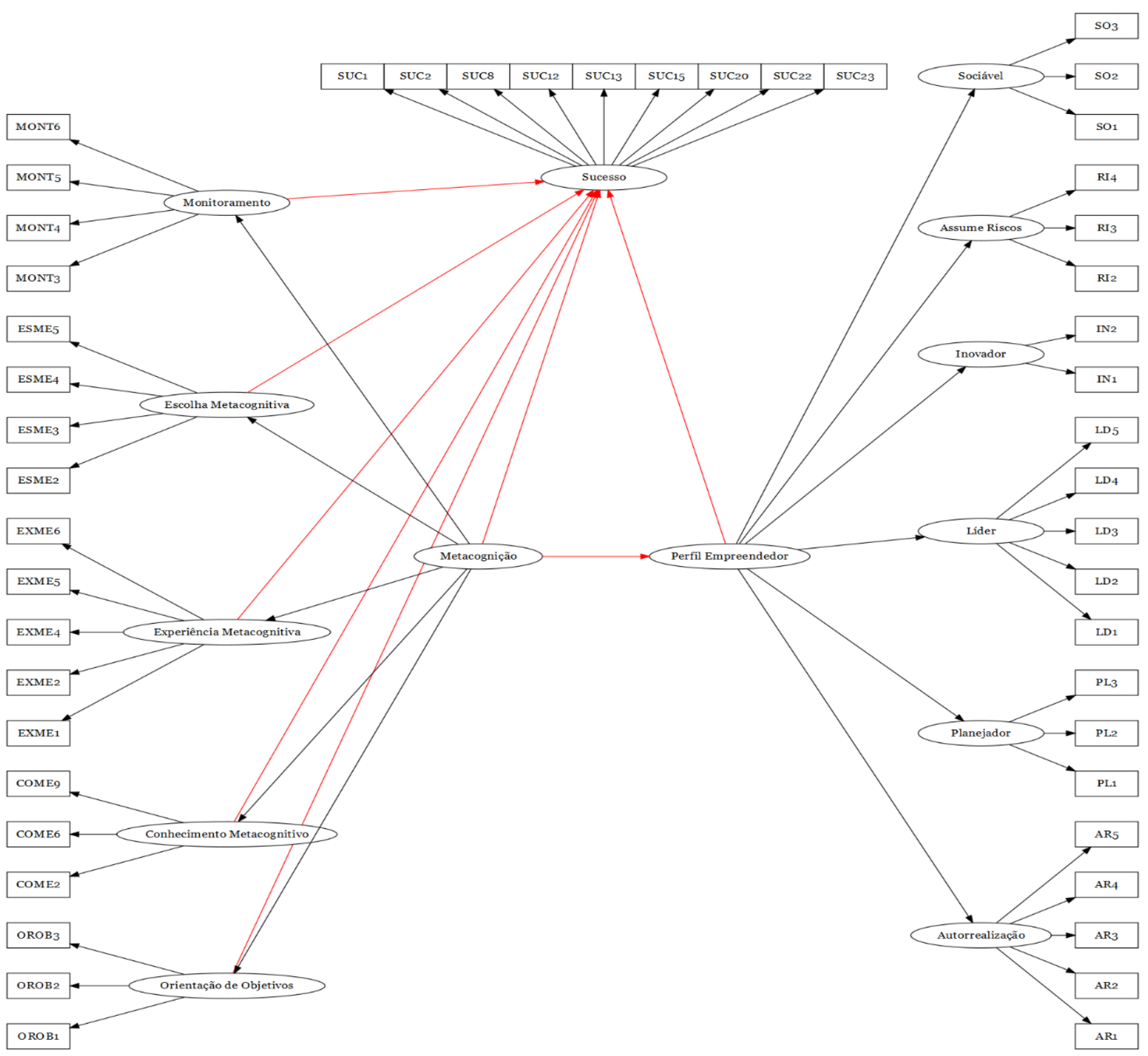

Figura 1. Modelo de mensuração final

Fonte: Elaborada pelo autor (2021). discriminante.

O modelo apresentou valores de HTMT inferiores a 0,9 , indicando validade

Na Tabela 1, são apresentados os valores para os pressupostos do modelo de mensuração. Nas dimensões autorrealização, inovador, líder, planejador e sociável, não foram excluídos itens e os pressupostos do modelo de mensuração foram atendidos. As dimensões conhecimento metacognitivo, escolha metacognitiva, experiência metacognitiva, monitoramento, orientação de objetivos, assume riscos e sucesso, devido a violações no pressuposto de validade convergente (AVE $<0,5$ e carga fatorial inferior a 0,4 ou 0,7 ), foi necessária a exclusão de alguns itens. $\mathrm{O}$ pressuposto de consistência interna $(0,6<\mathrm{CR}<0,9)$ não foi violado. 
Tabela 1

Avaliação dos pressupostos do Modelo de Mensuração

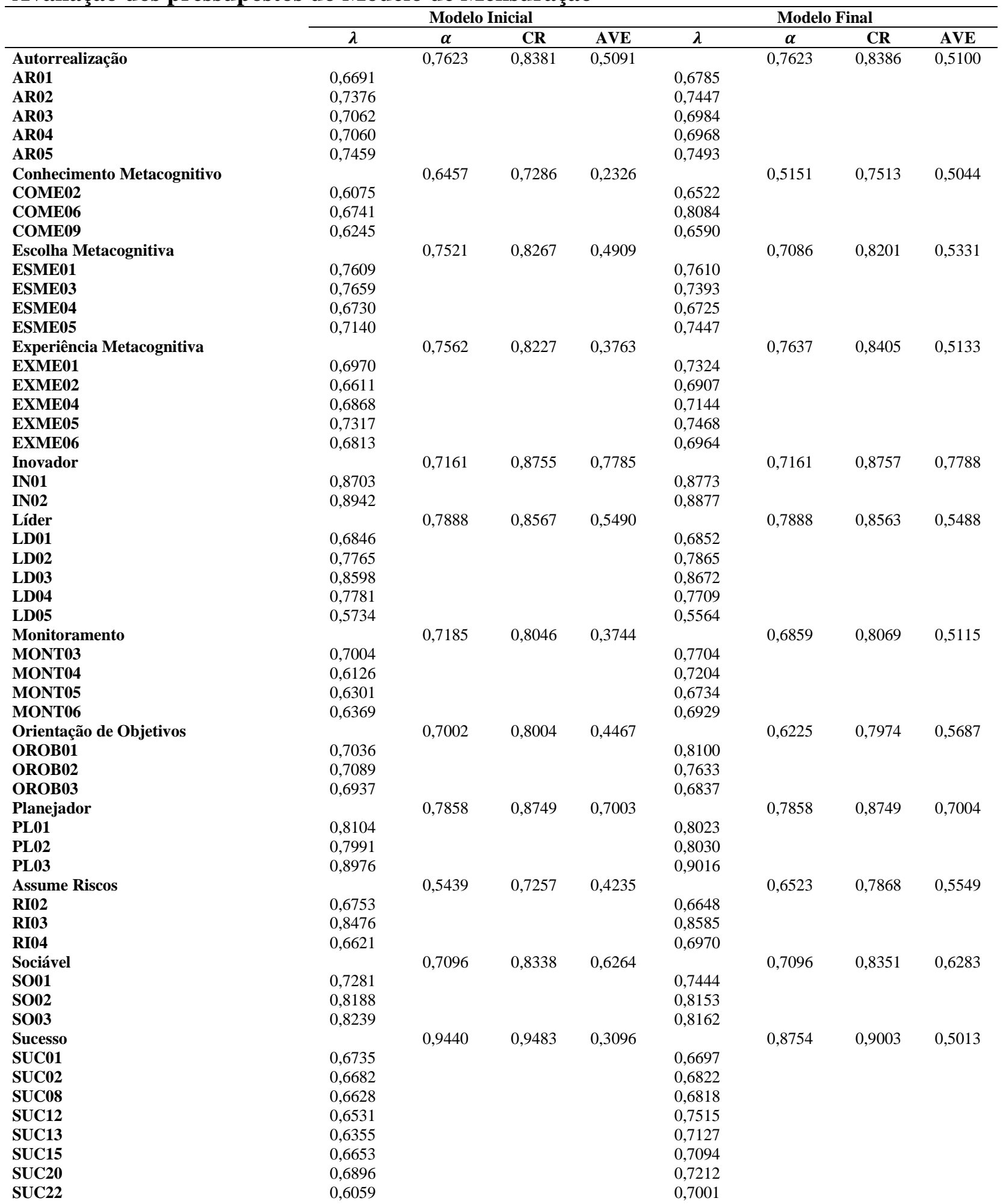

Legenda: $\lambda$ - carga fatorial. $\alpha$ - Alfa de Cronbach. CR - Composite Reliability. AVE - Average Variance Extracted.

Fonte: Elaborada pelo autor (2021). 
Além disso, como metacognição e perfil empreendedor não são dimensões medidas diretamente, propõe-se o uso de dimensões de segunda ordem, nível em que dimensões geram dimensões. Este estudo estimou o modelo do tipo reflexivo-reflexivo por meio do método de dois estágios (Sarstedt \& Cheah, 2019). Todo o procedimento proposto anteriormente descrito para validação da consistência dos constructos de segunda ordem deve ser repetido.

Em relação à validade discriminante, o modelo de segunda ordem apresentou valores de HTMT inferiores a 0,9, indicando existência de validade discriminante.

Na Tabela 2 são apresentados os valores obtidos para os pressupostos do modelo de mensuração. Nas dimensões metacognição e perfil empreendedor, não houve violações no pressuposto de validade convergente (AVE $<0,5$ e carga fatorial inferior a 0,4 ou 0,7$)$. $\mathrm{O}$ pressuposto de consistência interna $(0,6<\mathrm{CR}<0,9)$ também não foi violado.

Tabela 2

\begin{tabular}{lcccc}
\multicolumn{4}{c}{ Avaliação dos pressupostos do Modelo de Mensuração de Segunda ordem } & \\
\hline & $\boldsymbol{\lambda}$ & CR & AVE & $\boldsymbol{\alpha}$ \\
\hline Metacognição & 0,660 & 0,844 & 0,523 & 0,770 \\
Orientação de objetivos & 0,735 & & & \\
Conhecimento metacognitivo & 0,829 & & & \\
Experiência metacognitiva & 0,598 & & & \\
Escolha metacognitiva & 0,772 & & & \\
Monitoramento & & 0,855 & 0,503 & 0,795 \\
Perfil empreendedor & 0,835 & & & \\
Autorrealização & 0,711 & & & \\
Planejador & 0,869 & & & \\
Líder & 0,551 & & & \\
Inovador & 0,665 & & & \\
Assume riscos & 0,559 & & & \\
Sociável &
\end{tabular}

Legenda: $\lambda$ : carga fatorial; $\alpha$ : Alfa de Cronbach; CR: Composite Reliability; AVE: Average Variance Extracted. Fonte: Elaborada pelo autor (2021).

\section{RESULTADOS}

No intuito de determinar os efeitos diretos e indiretos, este estudo seguiu os passos propostos por Hair, Gabriel e Patel (2014). O primeiro passo, conforme Tabela 3, foi realizar a verificação do efeito direto entre a variável independente e a dependente, sem a inclusão da variável mediadora.

Os constructos orientação de objetivos, conhecimento metacognitivo, experiência metacognitiva e monitoramento apresentam relação direta significativa com a percepção de sucesso com $\mathrm{f}^{2}$ (tamanho de efeito) entre 0,021 e 0,101 , dando suporte à relação.

Portanto, das cinco primeiras hipóteses, quatro se confirmaram. Apenas a H4 não se confirmou. Logo, as confirmações das hipóteses H1, H2, H3 e H5 corroboraram os estudos de Pajares, Britner e Valiante (2000), Haynie e Shepherd (2009), Cho e Jung (2014) e Jones, Davis e Thomas (2017). Por conseguinte, o aperfeiçoamento e o aprendizado das dimensões orientação de objetivos, conhecimento metacognitivo, experiência metacognitiva e monitoramento têm efeito positivo sobre a percepção de sucesso.

Sobre a dimensão orientação de objetivos, houve efeito considerado pequeno (Cohen, 1988). O efeito direto apresentou um tamanho de efeito $\left(\mathrm{f}^{2}\right)$ de 0,082 e um nível de significância (p-valor) menor que 0,001 . Portanto, a orientação de objetivos tem relação direta significativa com a percepção de sucesso.

Em relação à dimensão conhecimento metacognitivo, também ficou demonstrado efeito direto significativo com a percepção de sucesso. Essa relação teve $\mathrm{f}^{2} \quad 0,101$, considerado 
pequeno, e um nível de significância menor que 0,001. Logo, o conhecimento metacognitivo apresenta relação direta significativa com a percepção de sucesso.

No que tange à experiência metacognitiva, existe um efeito significativo direto em sua relação com a percepção de sucesso. Essa relação demonstrou tamanho do efeito de 0,085 e nível de significância menor que 0,001 , dando suporte à hipótese H3. Desse modo, a experiência metacognitiva tem relação direta significativa com a percepção de sucesso. No entanto, o tamanho do efeito é considerado pequeno (Cohen, 1988).

O monitoramento metacognitivo também apresentou efeito direto significativo com a percepção de sucesso. Nessa relação, o tamanho do efeito foi de 0,021 e o nível de significância apresentou valor de 0,009, dando suporte à hipótese H5. Apesar de o efeito ser considerado pequeno, há uma relação direta significativa entre o monitoramento metacognitivo e a percepção de sucesso.

A escolha metacognitiva não apresentou efeito direto significativo com a variável dependente. O tamanho do efeito foi de 0,005 e o p-valor foi de 0,152. Nesse caso, não houve suporte para a hipótese $\mathrm{H} 4$.

Tabela 3

Efeito Direto entre as Dimensões Metacognitivas e Percepção de Sucesso

\begin{tabular}{|c|c|c|c|c|c|c|}
\hline Independente & Dependente & B & $\mathbf{f}^{2}$ & $\mathbf{T}$ & p-valor & Suporte \\
\hline Orientação de Objetivos & & 0,232 & 0,082 & 3,391 & $<0,001$ & Sim \\
\hline Conhecimento metacognitivo & & 0,268 & 0,101 & 3,701 & $<0,001$ & Sim \\
\hline Experiência Metacognitiva & Sucesso & 0,273 & 0,085 & 3,611 & $<0,001$ & Sim \\
\hline Escolha Metacognitiva & & 0,061 & 0,005 & 0,973 & 0,152 & Não \\
\hline Monitoramento & & 0,131 & 0,021 & 2,115 & 0,009 & Sim \\
\hline
\end{tabular}

Fonte: Elaborada pelo autor (2021).

O segundo passo foi avaliar o efeito da variável mediadora (perfil empreendedor) na relação entre a metacognição e a percepção de sucesso. A fim de avaliar a hipótese 6 , conforme Tabela 4, realizamos a verificação do efeito direto, indireto e total entre metacognição e percepção de sucesso mediada por perfil empreendedor.

Tabela 4

Modelo Estrutural de Segunda Ordem - Relação Direta

\begin{tabular}{lcccccc}
\hline Efeito & $\mathbf{B}$ & $\mathbf{f}^{\mathbf{2}}$ & $\mathbf{T}$ & $\mathbf{p - v a l o r}$ & $\mathbf{I C 9 5 \%}$ & Suporte \\
\hline Total & 0,705 & 0,676 & 19,409 & $\mathbf{< 0 , 0 0 0 1}$ & 0,$638 ; 0,780$ & Sim \\
Direto & 0,304 & 0,162 & 5,194 & $\mathbf{< 0 , 0 0 0 1}$ & 0,$184 ; 0,410$ & Sim \\
Indireto & 0,402 & 0,514 & 8,690 & $\mathbf{< 0 , 0 0 0 1}$ & 0,$324 ; 0,509$ & Sim \\
\hline
\end{tabular}

Fonte: Elaborada pelo autor (2021).

Podemos afirmar que a metacognição influencia a percepção de sucesso diretamente e essa relação é em parte mediada pelo perfil empreendedor. No que tange à relação direta, a metacognição apresentou tamanho do efeito de 0,162, considerado médio, e nível de significância menor do que 0,001. Já inserindo a variável mediadora perfil empreendedor, o tamanho do efeito foi considerado grande $(0,514)$ e o nível de significância menor do que 0,001 , dando suporte à hipótese H6. Portanto, a metacognição apresenta relação direta significativa com a percepção de sucesso e, inserindo a variável mediadora perfil empreendedor, há relação significativa indireta entre metacognição e percepção de sucesso. 


\section{CONSIDERAÇÕES FINAIS}

Esta pesquisa teve o objetivo de identificar se a metacognição tem relação significativa com a percepção de sucesso e se o perfil empreendedor tem papel mediador nessa relação. Foi aplicada a MEE-PLS para a validação do modelo e o teste de hipóteses.

Foram aplicados instrumentos psicométricos no intuito de mensurar três constructos: a) metacognição; b) perfil empreendedor; e c) percepção de sucesso. Avaliaram-se os pressupostos de consistência interna e validade convergente e discriminante, chegando a um modelo final. Após as exclusões dos itens não relevantes, permaneceram questões de todos os subitens de cada instrumento, que, por consequência, manteve todas as categorias no modelo.

Os resultados desta pesquisa mostram que a orientação de objetivos, conhecimento metacognitivo, experiência metacognitiva e monitoramento metacognitivo apresentam relação direta significativa com a percepção de sucesso, confirmando H1, H2, H3 e H5. Os resultados também demonstraram que a escolha metacognitiva não tem relação direta significativa com a percepção de sucesso, ou seja, a H4 não foi confirmada.

Utilizando o modelo de segunda ordem, este estudo demonstrou que a metacognição tem relação direta significativa com a percepção de sucesso e o tamanho do efeito é considerado médio (Cohen, 1988). Inserindo a variável mediadora, a relação entre metacognição e percepção de sucesso apresentou tamanho do efeito considerado grande, dando suporte à hipótese H6.

Este trabalho promove avanços nessa linha de pesquisa, apresentando novas evidências empíricas de que, de fato, existe relação entre metacognição e percepção de sucesso. Contribuise, também, por evidenciar as características metacognitivas que devem ser trabalhadas para melhorar a percepção de sucesso. Em relação à orientação de objetivos, devem-se aperfeiçoar características como definir frequentemente objetivos, compreender a relação entre tarefas e objetivos, estabelecer objetivos específicos e avaliar com frequência o progresso de uma tarefa. No que tange ao conhecimento metacognitivo, deve-se incentivar o aprendizado e o aperfeiçoamento de características como selecionar a melhor opção para a resolução de problemas, usar estratégias assertivas no passado, obter conhecimento prévio de uma tarefa, bem como fragmentar problemas em pequenas partes. Sobre a experiência metacognitiva, deve-se aprender e aperfeiçoar características como pensar antes de realizar uma tarefa, usar diferentes estratégias, organizar o tempo e informações, selecionar informações importantes e utilizar a intuição para formular estratégias. Já sobre o monitoramento, deve-se tentar aprimorar características como reavaliar informações, ter ciência das estratégias a ser utilizadas, analisar a utilidade de estratégias, checar a compreensão do problema e avaliar o êxito de uma nova tarefa.

Esta pesquisa ainda pode contribuir para estudos futuros como um instrumento de investigação científica relacionada à metacognição, a partir de um modelo conceitual, bem como relacionada ao perfil empreendedor e à percepção de sucesso, além de ser uma nova possibilidade para examinar a metacognição.

Este trabalho apresenta contribuições teóricas e práticas. As contribuições teóricas estão em oferecer referencial teórico atual sobre uma linha de pesquisa ainda carente no contexto nacional. No que tange às contribuições práticas, o trabalho oferece conclusões empíricas que poderão ajudar administradores, contadores, economistas, professores, instituições de ensino superior e profissionais a compreenderem a metacognição e seu impacto na percepção de sucesso.

\section{REFERÊNCIAS}

Botelho, P., \& Vargas, D. S. (2021). Inferências e atividades de leitura: cognição e metacognição em sala de aula. Cad. Est. Ling., 63, 1-14. 
Cheah, Jun-Hwa, Sarstedt, M., Ringle, C. M., Ramayah, T., \& Ting, H. (2018). Convergent validity assessment of formatively measured constructs in PLS-SEM: On using single-item versus multi-item measures in redundancy analyses. International Journal of Contemporary Hospitality Management, 30(11), 3192-3210.

Cho, Y. S., \& Jung, J. Y. (2014). The relationship between metacognition, entrepreneurial orientation, and firm performance: an empirical investigation. Academy of Entrepreneurship Journal, 20(2), 71-84.

Cohen, J. (1988). Statistical Power Analysis for the Behavioral Sciences (2a.ed.). Hillsdale, NJ: Lawrence Erlbaum Associates.

Costa, L. V., \& Dutra, J. (2011). Avaliação da carreira no mundo contemporâneo: proposta de um modelo de três dimensões. Recape - Revista de Carreiras e Pessoas, 1(01), 1-22.

Costa, L. V. (2013). Construção e Validação de uma Escala de Percepção de Sucesso na Carreira. Revista RECAPE, 3(1).

Deffendi, L. T., \& Schelini, P. W. (2016). O monitoramento metacognitivo em tarefas que envolvem a criatividade verbal. Psicologia: Teoria e Pesquisa, 32(3),1-8.

Dijkstra, T. K., \& Henseler, J. (2015). Consistent partial least squares path modeling. MIS quarterly, 39(2).

Dries, N., Pepermans, R., \& Carlier, O. (2008). Career success: Constructing a multidimensional model. Journal of Vocational Behavior, 73(2), 254-267.

Earley, P. C. \& Ang, S. (2003). Cultura intelligence: individuals interections across cultures. Stanford, Califórnia: Stanford Business Books.

Estrela, P. M. C. de A. (2020). Metacognição, Perfil Empreendedor e Percepção de Sucesso. Tese de doutorado, Universidade Federal da Bahia, Salvador, BA, Brasil.

Ferreira, M. P. V., Pinto, C. F., \& Miranda, R. M. (2015). Três décadas de pesquisa em empreendedorismo: uma revisão dos principais periódicos internacionais de empreendedorismo. READ. Rev. eletrôn. adm., 21(2), 406-436.

Ferreira, M. A., Colares, A. C. V, Rocha, P. M., \& Carvalho Junior, L. E. (2013). O Ensino de Empreendedorismo nos Cursos de Graduação em Ciências Contábeis. Revista Catarinense da Ciência Contábil, 12(35), 63-78.

Flavell, J. H. (1987). Speculations about the nature and development of metacognition. Hillside, NJ: Erlbaum.

Flavell, J. H. (1979). Metacognition and cognitive monitoring: A new area of cognitivedevelopmental inquiry. American Psychologist, 34(10), 906-911. 
Gewehr, D., Strohschoen, A. A. G., \& Schuck, R. J. (2020). Projetos de pesquiisa e a relação com a metacognição: percepções de alunos pesquisadores sobre a própria aprendizagem. Revista Ensaio, 22.

Hair, J. F., Hult G. T. M., Ringle, C. M., Sarstedt, M., \& Thiele, K. O. (2017). Mirror, mirror on the wall: a comparative evaluation of composite-based structural equation modeling methods, Journal of the Academy of Marketing Science, Springer, 45(5), 616-632.

Hair, J. F., GabrieL, M. L. D. S., \& Patel, V. K. (2014). Modelagem de equações estruturais baseado em covariância (CB-SEM) com o AMOS: orientações sobre a sua aplicação como uma ferramenta de pesquisa de marketing. REMark-Revista Brasileira de Marketing, edição especial, 13(2), 44-55.

Haynie, M., \& Shepherd, D. A. (2009). A Measure of Adaptive Cognition for Entrepreneurship Research. Entrepreneurship Theory and Practice, 33(3), 695-714.

Haynie, J. M., Shepherd, D., Mosakowski, E., \& Earley, P. C. (2010). A situated metacognitive model of the entrepreneurial mindset. Journal of Business Venturing, 25(2), 217-229.

Henseler, J., Ringle, C. M., \& Sarstedt, M. (2015). A new criterion for assessing discriminant validity in variance-based structural equation modeling. Journal of the academy of marketing science, 43(1), 115-135.

Hirst, G., Knippenberg, D. V., \& Zhou, J. (2009). A cross-level perspective on employee creativity: goal orientation, team learning behavior, and individual creativity. Academy of Management Journal, 52(2), 280-293.

Jones, J. L., Davis, W. D., \& Thomas, C. H. (2017). In competition engaging? Examining the interactive effects of goal orientation and competitive work environment on engangement. Human Resource Management, 56(3), 389-405, 2017.

Kock, N., \& Hadaya, P. (2016). Minimum sample size estimation in PLS-SEM: The inverse square root and gamma-exponential methods. Information Systems Journal, 28(1), 227-261.

Lima Filho, R. N. (2013). Empreendendo sobre o empreender e pensando sobre o pensar: um estudo sobre características empreendedoras e metacognição. (Tese de doutorado), Universidade Federal da Bahia, Salvador, BA, Brasil.

Lima Filho, R. N., \& Bruni, A. L. (2015). Metacognição estimula características empreendedoras? Uma análise em profissionais de administração. RACE, 14(2), 427-450.

Lima Filho, R. N., \& Nova, S. P. C. C. (2020). Diferenças de aprendizagem autorregulada em estudantes de pós-graduação: uma análise relacionando idade e gênero ao uso de estratégias. Revista Catarinense da Ciência Contábil, 19, 1-20.

Long, J. S. (1983). Confirmatory Factory Analysis: a prefacy to LISREL. Sage Publications, $7(33)$. 
Moreira, M. A., Alves, N. J. F., Andreassi, T., \& Braga, J. G. R. (2020). Educação Empreendedora em Contabilidade: da Teoria à Aprendizagem Experiencial. Revista Catarinense da Ciência Contábil, 19(1).

Oosterbeek, H., Praag, M. V., \& Ijsselstein, A. (2010). The impact of entrepreneurship education on entrepreneurship skills and motivation. European Economic Review, 54(1), 442-454.

Pajares, F., Britner, S., \& Valiante, G. (2000). Relation between achievement goals and selfbeliefs of middleschool students in writing and science. Contemporary Educational Psychology, 25, 406-422.

Pimentel, F. S. C., \& Sales Júnior, V. B. de (2021). Avaliação da consciência metacognitiva de usuários de jogos digitais. Aprendizagem baseada em jogos digitais: teoria e prática. Rio de Janeiro: BG Business Graphics Editora.

Reijonen, H., \& Komppula, R. (2007). Perception of success and its effect is small firm performance. Journal of Small Business and Enterprise Development, Finland, 14(4).

Ribeiro, C. (2003), Metacognição: um apoio ao processo de aprendizagem. Psicologia: Reflexão e Crítica, 16(1), 109-116.

Rocha, C. J. T., \& Malheiro, J. M. S. (2019). Metacognição e a experimentação investigativa: a construção de categorias interativa dialógicas. Revista Educação, 44.

Rosa, C. T. W., Corrêa, N. N. G., Passos, M. M., \& Arruda, S. de M. (2020). Metacognição e seus 50 anos: uma breve história da evolução do conceito. Revista Educar Mais, 4(3), 703721.

Sarstedt, M., \& Cheah, J. H. (2019). Partial least squares structural equation modeling using SmartPLS: a software review. Journal of Marketing Analytics, 196-202, 2019.

Schmidt, S., \& Bohnenberger, M. C. (2009). Perfil empreendedor e desempenho organizacional. Revista de Administração Contemporânea, 13(3) 450-467.

Staniewsky, M. W., \& Awruk, K. (2017). Questionnaire of entrepreneurial success: report on inicial stage of method construction. Journal of Business Research.

Urban, B. (2012). Applying a metacognitive perspective to entrepreneurship: empirical evidence on the influence of metacognitive dimensions on entrepreneurialintentions. Jornal of Enterprising Culture, 20(2), 203-225. 